\title{
Atlas de Riesgos de la Isla de Cozumel, México
}

Recibido: 05/20/2016 · Aceptado: 15/03/2016

Oscar Frausto Martínez* Laboratorio de Observación e Investigación Espacial (UQROo) REDESClim

Thomas Ihl Facultad de Geografía Universidad de Halle-Wittenberg Justo Rojas López Unidad de Género Instituto Politécnico Nacional

\section{Resumen}

El atlas muestra de manera sintética aquellos peligros naturales que podrían generar desastre en la isla de Cozumel, Quintana Roo, México. A través de un análisis espacial del tipo de amenazas (naturales y antrópicas) y la vinculación con la vulnerabilidad social, en el nivel de manzana y lote, se determina el riesgo a diversos fenómenos, entre ellos las inundaciones, la erosión costera, las lluvias extremas, las marejadas de tormenta y los hundimientos diferenciales del terreno. Se identifican las viviendas en extrema pobreza y que son las de mayor vulnerabilidad. El aporte principal de este estudio se centra en la generación de un banco de datos geo-referenciado de los peligros, el mapeo de las condiciones de vulnerabilidad y los escenarios de riesgo, lo que permitirá el monitoreo y la actualización de la información base.

Palabras clave: Resiliencia, georreferencia, gestión de la reducción del riesgo, desastres.

*Correo electrónico: ofrausto@uqroo.edu.mx 


\title{
Risk Atlas of Cozumel Island, Mexico
}

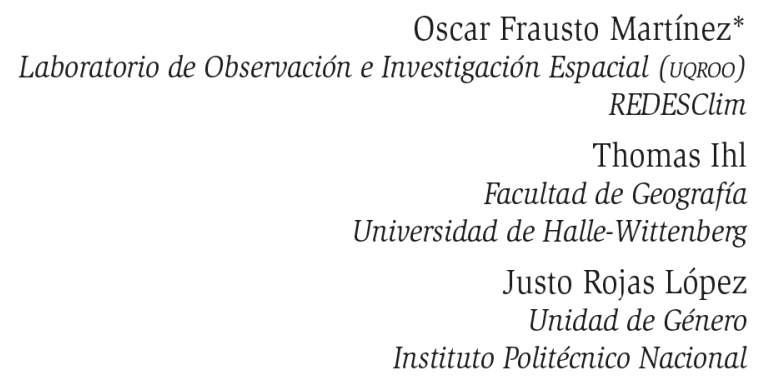

\begin{abstract}
The atlas shows the potential natural hazard, which can cause at any time and any place on the island of Cozumel a disaster. Threats, whether natural or anthropogenic kind, will be analyzed and linked with social vulnerability, either on a building block level or on land parcels. The risk is fixed to certain phenomena, such as inundation, coastal erosion, extreme precipitation, storm surges and differential subsidence of the land. Dwellings in extreme poverty present households with major vulnerability. The main contribution of this study focuses on a compilation of a georeferenced database of hazards, risk situations and vulnerability mapping, which allow monitoring the threat and updating of the original database.
\end{abstract}

KEY worDs: Resilience, georeferences, risk management, disasters.

*E-mail: ofrausto@uqroo.edu.mx 


\section{Introducción}

El objetivo de este artículo es señalar los principios teóricos y técnicos que fundamentan el Atlas de Riesgos de la Isla de Cozumel. Se parte de los alcances del primer ejercicio en su tipo desarrollado en 2004, previo a las inundaciones provocadas por los huracanes Emily y Wilma, en julio y octubre de 2005, con repercusiones negativas en las localidades del noreste de la Península de Yucatán.

El Atlas de Riesgos de la Isla de Cozumel de 2004 no cumplió las expectativas en torno a la reducción del riesgo ante los efectos de los huracanes de 2005, por lo que se propone constituir una actualización del Atlas bajo nuevas directrices, a saber:

- Los principios de aprendizaje que dejó la experiencia en la gestión de los huracanes Emily y Wilma.

- La conceptualización base de la gestión de la reducción del riesgo ante desastres.

- Reconocimiento de la experiencia local para hacer frente a las amenazas, reducir la vulnerabilidad y ampliar su resiliencia.

- Que la información del Atlas pueda actualizarse continuamente.

Así, por medio de la exposición de los principios de elaboración, los métodos y materiales, además de unos ejemplos, se presentan los alcances del nuevo Atlas de Riesgos de la Isla de Cozumel, actualizado en 2012, y aprobado por el cabildo de Cozumel y el Centro Nacional de Prevención de Desastres (Cenapred) en 2014.

\section{Principios de elaboración del Atlas de Riesgos}

Un atlas es una herramienta para tomar decisiones; compila, muestra y señala dónde, cómo y qué sobre un fenómeno específico (Ihl, Frausto, Tun, Izquierdo y Rolfes, 2009, p. 96). A su vez, permite establecer zonas con diferente vocación o aptitud preferente, en función de los elementos antrópicos amenazados y los potencialmente afectables en el futuro (Palacio, 2014, p. 50). Los atlas de riesgo tienen como base dos componentes centrales (Shi, Wang, Liu, Zhang, Chen y Lv, 2009): 
a) el fundamento teórico de organización, y

b) las técnicas utilizadas para la generación de los cartogramas.

El fundamento teórico se describe en la aproximación epistemológica, donde la comunidad o la sociedad será determinante (Frerks, Warner y Weijs, 2011, p. 106). Bajo este principio, los atlas tendrán un componente singular de acuerdo con el territorio que se gestione, la escala o jurisdicción espacial.

Conjuntamente, los apartados o secciones están definidos por el marco de referencia teórico, donde la infraestructura es el referente de seguridad para la sección de los peligros; los grupos sociales, la comunidad (urbana, agrícola, industrial-densa, pobre), para señalar la reducción de probabilidad e impacto, así como el incremento de las capacidades para hacerlos menos vulnerables; el terreno y su uso (inundable, sísmico o hundimiento), para disminuir la probabilidad de riesgo. En la actualidad, desde una aproximación sistémica, se busca aumentar la adaptación bajo el principio de resiliencia (Birkmann, 2006; Hernández-Aguilar y Castillo-Villanueva, 2012; Castillo-Villanueva y VelázquezTorres, 2015).

Por el referente técnico, los mapas generados dependerán de la resolución espacial de la información, las bases de datos georreferenciadas y la sistematización de la información a representar (estático-dinámico, histórico o de pronóstico, papel-virtual) (Shi et al., 2009; Frausto, Ihl, Rojas y Morales, 2014).

Así, de acuerdo con las premisas anteriores, se entiende que un atlas es "una colección de mapas o documentos cartografiados que se preparan especialmente para brindar información global o lo más completa posible sobre fenómenos geográficos en un espacio determinado" (Aadlers y Crane cit. en Ihl et al., 2009, p. 95). En el ámbito específico, la Ley General de Protección Civil considera el atlas de riesgo como un instrumento para la planificación del uso del suelo (Sánchez y Cavazos, 2015; Sedatu, 2014).

El Atlas de Riesgos del Municipio de Cozumel se origina a partir del proyecto de investigación Bases de Datos Georreferenciadas de Desastres y Fenómenos Hidrometeorológicos Extremos de la Península de Yucatán, con apoyo de la Universidad de Quintana Roo, el Ayuntamiento Municipal de Cozumel y el programa de la Secretaría de Desarrollo Social (Sedesol) Hábitat 2011 (H. Ayuntamiento de Cozumel, 2012). 


\section{Área de estudio}

El área de estudio comprende la totalidad de la isla de Cozumel, ubicada en el mar Caribe, a $18 \mathrm{~km}$ de la costa noreste de Quintana Roo (figura 1), con más de 86000 habitantes para 2015 (INEGI, 2015) y puerto de entrada de más de dos millones de turistas de cruceros al año (Mota y Frausto, 2015). La población se concentra en el sector occidental de la isla, la cual cuenta con áreas protegidas en las que se ofertan servicios de recreación para los turistas.

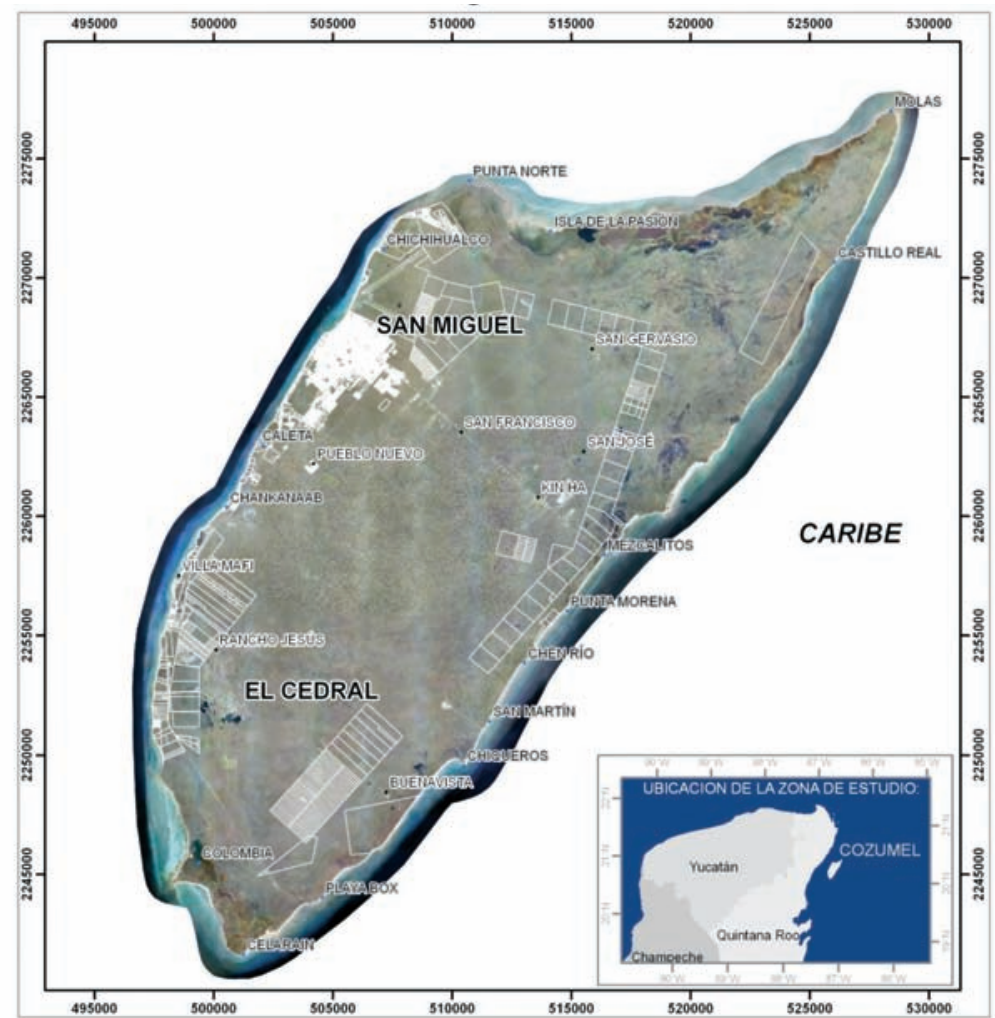

Ubicación: Isla de Cozumel

Fuente: Elaboración propia.

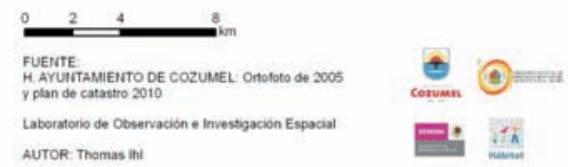

Figura 1. Mapa de ubicación de la isla de Cozumel 


\section{Métodos y materiales}

Para la realización del Atlas de Riesgos de la Isla de Cozumel se parte de las metodologías propuestas por el Cenapred, en su serie de libros Guía Básica para la Elaboración de Atlas Estatales y Municipales de Peligros y Riesgos. Por ser un proyecto que debe de cumplir con las Reglas de Operación para su ejecución se cubren los lineamientos mínimos para la aprobación (considerando que el proyecto del Atlas se desarrolló en 2013), sin embargo, la mayoría de las metodologías fueron mejoradas, adaptadas al ambiente insular y priorizando las amenazas a la infraestructura y la población de Cozumel, resultando una caracterización para el contexto local (los elementos de mejora se exponen en los apartados de Resultados y Discusión).

Los métodos utilizados se dividen en:

a) Identificación y caracterización de las amenazas. Se parte del sistema de reconocimiento realizado en 2004 (H. Ayuntamiento de Cozumel, 2004) y del histórico de daños provocados por los huracanes Emily y Wilma (Vivas, 2006).

b) Reconocimiento de los peligros de acuerdo con su diferenciación genética: geológicos (fallas y fracturas; hundimientos, derrumbes y deslizamientos; erosión); hidrometeorológicos (ciclones, tormentas eléctricas, sequías, temperaturas extremas, vientos fuertes, inundaciones y masas de aire) y otras amenazas asociadas (explosiones e incendios), y antropogénicos o tecnológicos. En este documento solo se muestran ejemplos de los mapas de inundaciones pluviales y erosión costera, por ser las de mayor relevancia en el contexto insular. La resolución de los datos espaciales se divide en tres. Primero, para la descripción de los mapas generales se construyeron a escala 1:250000 (considerando las bases generales del Instituto Nacional de Estadística y Geografía, INEGI). Segundo, para los mapas de peligros, la base de información fue a 1:4500 para el caso de la mancha urbana, y la zona conurbada de 1:7500 a 1:10000. Tercero, respecto a hundimientos, erosión, inundaciones, fallas y fracturas, y marea de tormenta, la información se derivó de los modelos del terreno de datos LiDAR (datos de $0.73 \mathrm{~cm}$ de resolución) (Colín, Chimal e Ihl, 2015; Colín, Frausto, Jiménez y García, 2015). Los datos de clima se 
construyeron a partir de los modelos generales del Atlas Climático Digital de México, con mallas de resolución de 9 x 9 km (Fernández, Romero y Zavala, 2014), a fin de enriquecer la información puntual de la única estación climática con la que se cuenta en el territorio insular de Cozumel.

c) En la sección de vulnerabilidad, se considera la definición de Rojas y Frausto (2014), quienes señalan que la vulnerabilidad social a peligros debe estar conectada con las características sociales que retan estos fenómenos naturales. Tales factores, tanto los de resiliencia como los de resistencia, están vinculados principalmente con la calidad de la vivienda, el acceso a servicios de salubridad y el nivel educativo. Es decir, que son estos factores lo que harían a los seres humanos capaces de recuperarse ante una amenaza como la mencionada. Así, se procedió a un análisis de componentes principales con el objetivo de tener un índice de vulnerabilidad. Las variables resultantes del método de reducción fueron las siguientes: viviendas con piso de tierra, viviendas con hacinamiento y nivel educativo. A fin de enriquecer el índice de vulnerabilidad, se complementó esta sección con la densidad de población y el índice de marginación. La información se calculó en el nivel de manzana con los datos del Censo de Población y Vivienda 2010 (INEGI, 2010). En el sistema de información, se complementa el mapeo con información de construcción en el nivel de lote. Además, fueron añadidos otros elementos al sistema de vulnerabilidad, como vías de comunicación, muelles internacionales, aeropuertos, gasoductos, sistemas de drenaje y agua potable (Dirección de Desarrollo Urbano, 2010).

b) Aplicación del sistema de álgebra de mapas para reconocer la distribución de aquellos territorios en riesgo a desastres por peligros identificados. Se realizaron modelos de riesgo para tres temas y para 20 escenarios (en función de la génesis del fenómeno y los índices de vulnerabilidad, destacando magnitud, frecuencia, distribución espacial y, en su caso, temporal). 


\section{Resultados}

A continuación se presentan algunos ejemplos de los documentos generados en cada una de las secciones del Atlas; los elementos se centran en el fundamento conceptual y técnico de representación, destacando la resolución de las bases de datos y mejora respecto al Atlas de 2004.

Por su ubicación, la recurrencia de eventos como tormentas tropicales, huracanes, nortes, sequías y precipitaciones, además de inundaciones, procesos de erosión costera y marejadas de tormentas, el Atlas tiene un componente hidrometeorológico de gran peso. Así, se muestran dos mapas capitales para la toma de decisiones que los gestores municipales (del Comité de Protección Civil) solicitaron realizar en calidad de "prioritarios y fundamentales".

\section{Amenazas por inundaciones y marejadas de tormentas}

Las inundaciones, muy habituales en la isla de Cozumel, son resultado de intensas precipitaciones y de un drenaje deficiente producto del sustrato calizo, con baja permeabilidad. Durante los meses lluviosos, de junio a noviembre, o en episodios de fuertes lluvias, se han originado encharcamientos e inundaciones recurrentes. Así, a través del reporte de zonas inundadas durante los huracanes de 2005, la modelación de depresiones topográficas y el reporte de inundaciones a Protección Civil, se generó un mapa de zonas con inundaciones parciales y frecuentemente inundadas (figura 2). Su escala de resolución es de 1:4500, base de ortofoto del INEGI de 2008; los datos de inundación se han modelado con base en datos puntuales de los reportes señalados.

Para el mapa de amenaza por oleaje de tormentas (figura 3), se parte de las afectaciones provocadas por la marejada y daños registrados después del huracán Wilma de 2005; la escala de resolución es a nivel de metros de distancia a la costa y por edificio o construcción. Se realiza para el litoral urbano, a lo largo del malecón de la ciudad de San Miguel de Cozumel. El sistema de edificaciones se configuró a partir del levantamiento catastral de 2008 y se sistematizó con los datos del modelo digital de elevación derivado del modelo superficial LiDAR (INEGI, 2009). La escala de resolución de este mapa es a 1:4500, base de ortofoto del INEGI de 2008 (INEGI, 2008); los daños son puntuales en el nivel de lote-edificación. 


\section{Atlas de Riesgos Cozumel 2011}

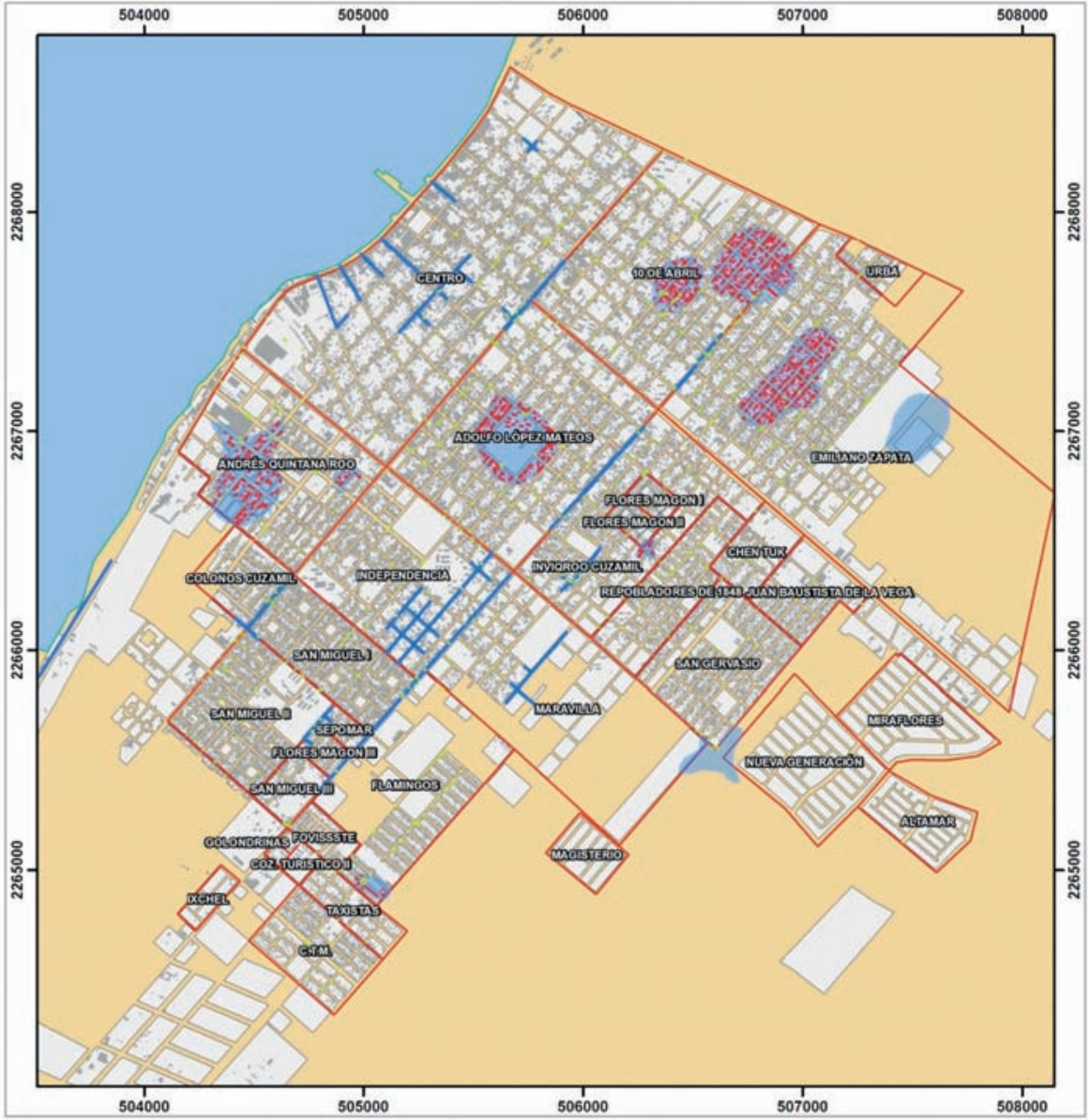

Mapas de Riesgo: Áreas frecuentemente inundadas

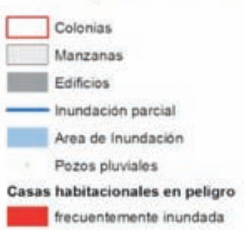

e inundaciones parciales
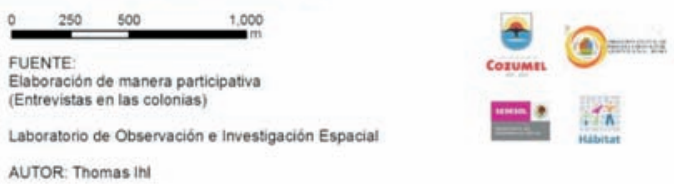

Fuente: Elaboración propia.

Figura 2. Mapa de áreas frecuentemente y parcialmente inundadas 


\section{Atlas de Riesgos Cozumel 2011}

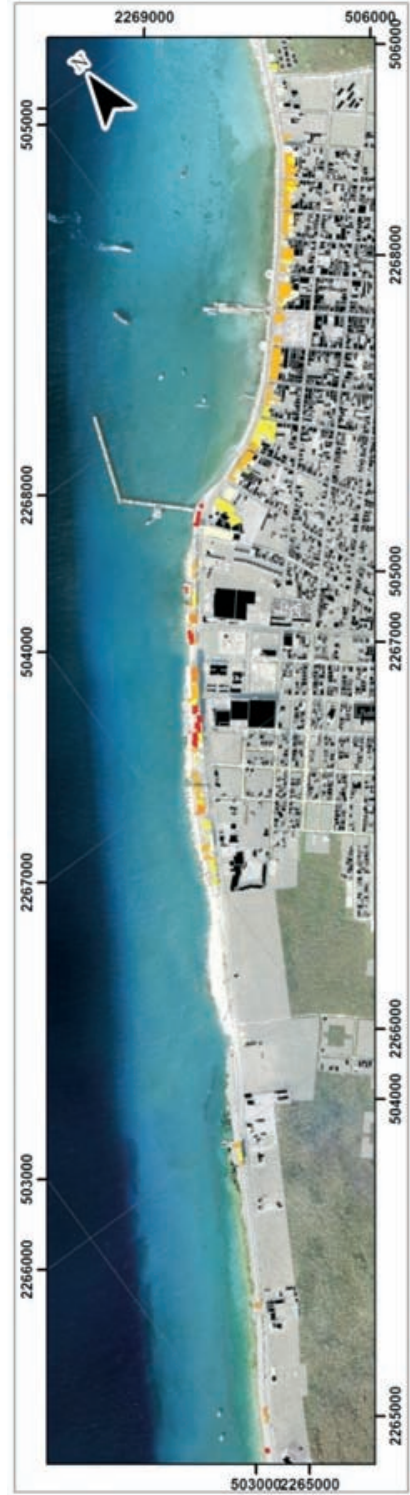

Mapa de Riesgo:

Destrucción de edificios por los oleajes de tormenta

\section{Riesgo por oleajes}
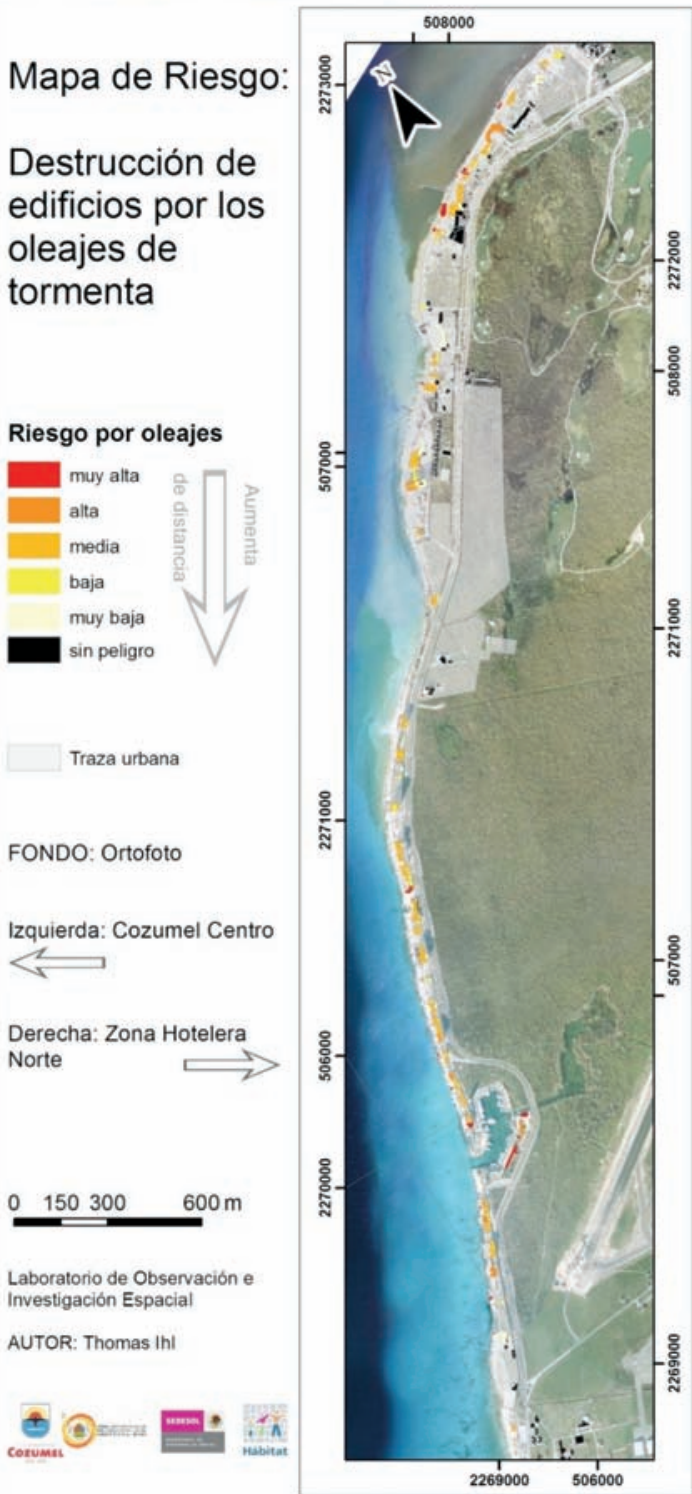

Fuente: Elaboración propia.

\section{FONDO: Ortofoto}

Izquierda: Cozumel Centro

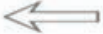

Derecha: Zona Hotelera

Norte
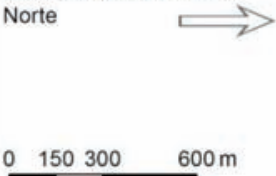

Laboratorio de Observación e Investigación Espacial AUTOR: Thomas |h|

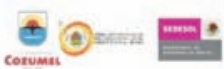
$\therefore$

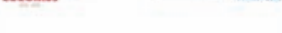

Figura 3. Mapa de construcciones amenazadas por oleaje de tormentas 


\section{Vulnerabilidad social}

Los elementos que construyen un índice de vulnerabilidad social a inundaciones y huracanes deben estar conectados con las características sociales que retan estos fenómenos naturales. Tales factores, tanto los de resiliencia como los de resistencia, están vinculados principalmente con la calidad de la vivienda, el acceso a servicios de salubridad y el nivel educativo. Es decir, son estos factores los que harían a los seres humanos capaces de resistir y de recuperarse de una amenaza como la apuntada. Se utiliza para ello el método de componentes principales, el cual reduce de un conjunto de variables probadas, las que, entre sí, tienen más cohesión y, por lo tanto, en grupo se pueden trabajar entrelazadas, descartando repeticiones y traslapes. Entonces, las variables que fueron consistentes en las pruebas han sido las que se tomaron para crear el índice de vulnerabilidad social.

Las variables resultantes del método de reducción fueron: viviendas con piso de tierra, viviendas con hacinamiento y nivel educativo. La resolución espacial fue a nivel manzana de la ciudad, siempre y cuando existiesen datos disponibles. De esta manera, para las viviendas con piso de tierra se calculó el porcentaje de estas para cada una de las manzanas de la ciudad; en cuanto a las viviendas con hacinamiento, se obtuvo el porcentaje de viviendas con tres o más personas por dormitorio, descontando baños y cocinas, donde no se duerme; se empleó como variable el número de personas por habitación. Finalmente, respecto al nivel educativo, se consideró el porcentaje de la población de 15 años y más sin secundaria completa; el indicador se elaboró con los siguientes datos: a) población de 15 años o más sin instrucción; b) población de 15 años o más con primaria completa; c) población de 15 años o más con estudios técnicos o comerciales con primaria terminada; d) población de 15 años o más que aprobó entre el primer y el segundo año de secundaria; e) población de 15 años o más que no especificó su grado aprobado en secundaria, y f) población de 15 años o más que no especificó su nivel de instrucción.

Además de estos factores, que forman parte del índice de vulnerabilidad social, se calcularon la densidad poblacional y el índice de marginación. Para la primera se calculó el número de habitantes en las manzanas y el total en metros cuadrados que mide cada manzana con lo cual se obtuvo el cociente de habitantes por metro cuadrado. En tanto que para el segundo se reunieron variables como salud, educación, fallecimiento de hijos, y viviendas sin refrigerador, a 


\section{Atlas de Riesgos Cozumel 2011}

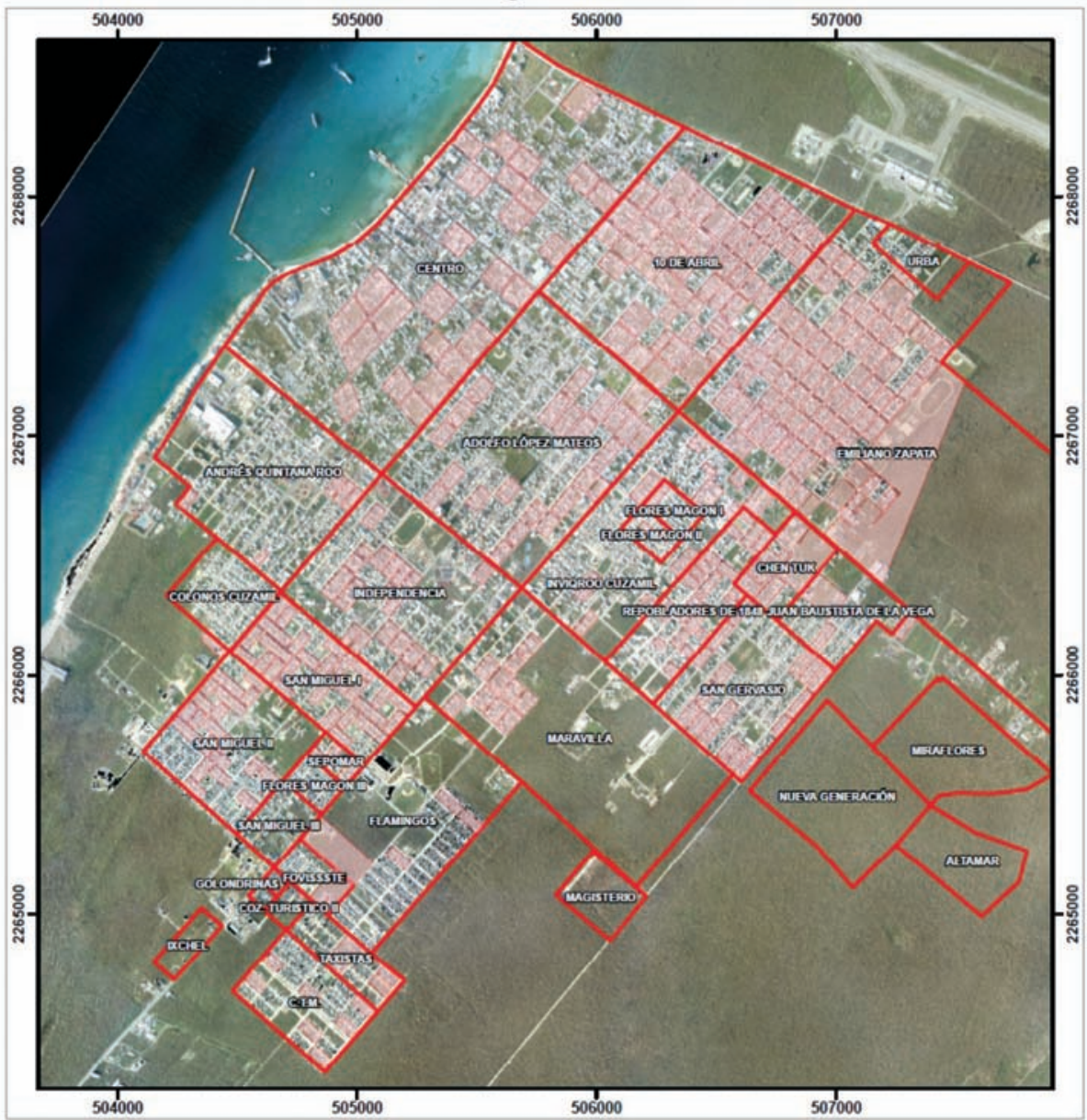

\section{Mapas sociales: Vulnerabilidad social}

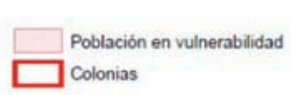

Colonias
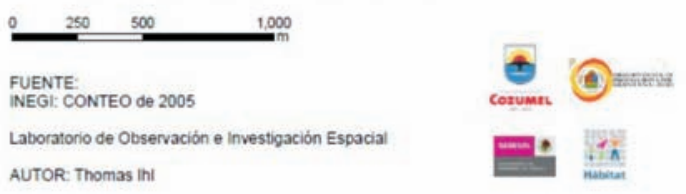

Fuente: Elaboración propia.

Figura 4. Mapa de vulnerabilidad social 
Atlas de Riesgos Cozumel 2011

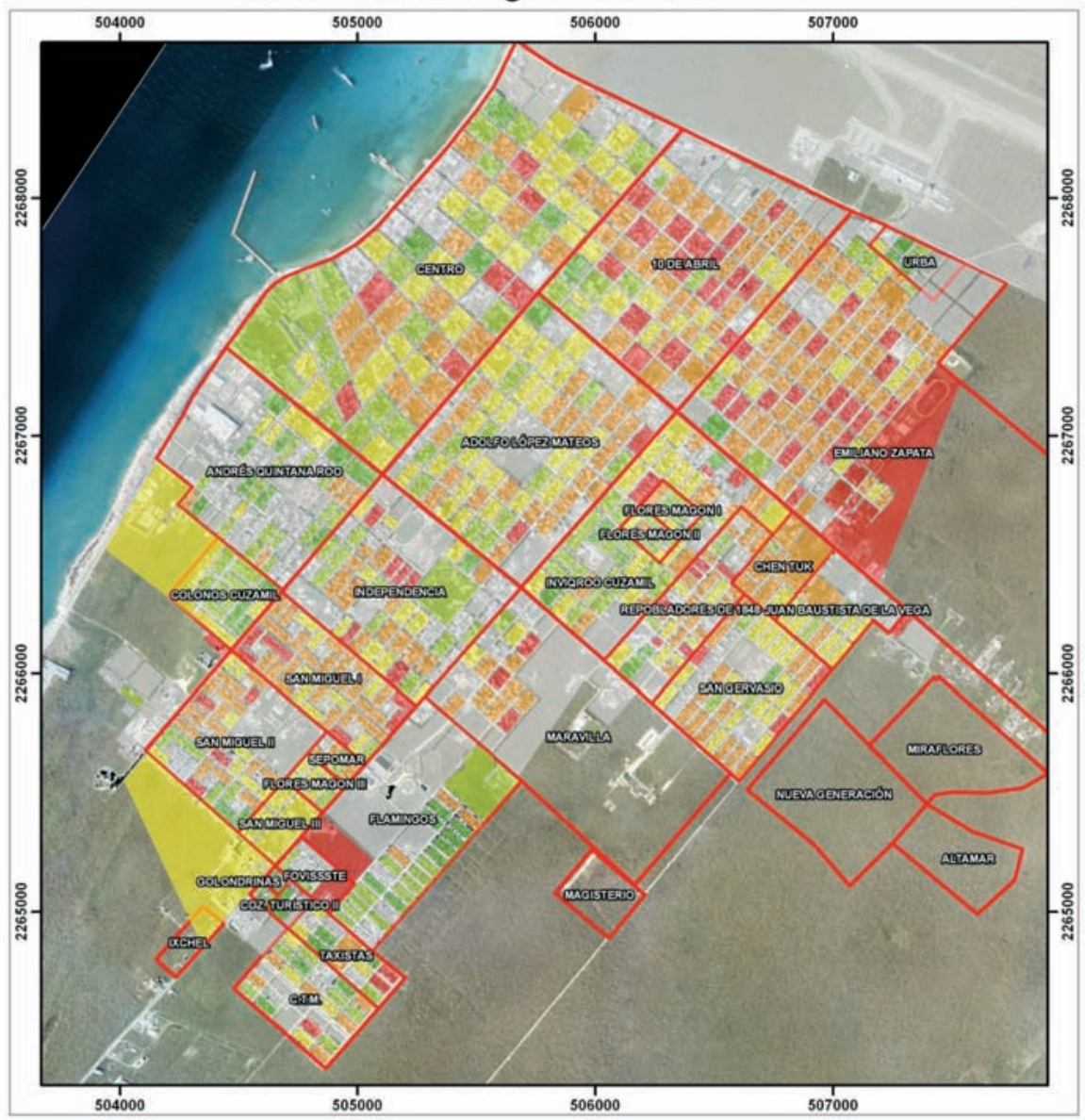

\section{Mapas sociales: Marginación}

Marginación
Niveles
sin datos o sin población
Muy bajo
Bajo
Medio
Alto
Muy alto
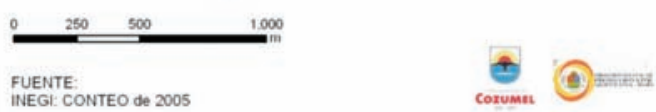

Fuente: Elaboración propia.

Figura 5. Mapa de marginación social a nivel manzana 
fin de construir un índice que mostró los diferentes matices del bienestar en la ciudad.

Así, para la elaboración del índice de vulnerabilidad social se utilizó la misma herramienta estadística de los componentes principales. En este caso, el resultado de los componentes principales arrojó un factor que cubría más de 50 \% de la variabilidad del conjunto de datos, lo cual refuerza su validez.

De tal suerte, mediante los mapas de vulnerabilidad social es posible presentar un resumen de los problemas del bienestar en la ciudad y, con el propósito de fortalecer la definición de unidades vulnerables, se consideró el instrumento de planificación local que es representado por el índice de marginación. Las figuras 4 y 5 exhiben los mapas de vulnerabilidad social y marginación, respectivamente.

\section{Discusión}

El Atlas de Riesgos de la Isla de Cozumel sigue los lineamientos de la Ley General de Protección Civil; si bien se cumple con los términos de referencia para su elaboración, estos no son suficientes o no son considerados para la planificación de los municipios o ciudades; asimismo, debido a la información de cohorte (2004, 2005, 2008 y 2011), se encuentra desactualizado, lo cual no permite una identificación de zonas de riesgo (Sánchez y Cavazos, 2015; HernándezAguilar y Castillo-Villanueva, 2012).

Además, debido a que el diagnóstico se realiza exclusivamente para las áreas reconocidas en el Plan de Desarrollo Urbano o Municipal, los asentamientos irregulares quedan fuera del sistema de acción de prevención ante riesgos, lo que se identifica como zonas "grises" o "inexistentes". Las áreas que no fueron integradas al Atlas se observan en la figura 6 y representan a más de 237 familias y 1500 habitantes (Ruiz, Frausto y Velázquez, 2015).

Dentro de los elementos más significativos del estudio está el relevamiento de información sobre las viviendas endebles en la mancha urbana de San Miguel de Cozumel; su ubicación y caracterización permitió su inclusión dentro de los programas de mejoramiento del hábitat y vivienda de 2012 a 2015. La localización de las viviendas endebles se puede apreciar en la figura 7. Lo anterior constituye una propuesta de conducción de los productos derivados del Atlas de Riesgos como instrumento para el desarrollo de programas públicos 
de mitigación o reducción de la vulnerabilidad, donde el fortalecimiento de las técnicas es fundamental (Hernández-Aguilar y Castillo-Villanueva, 2012).

Si bien el Atlas sirve para la planificación del territorio y mitigación de los desastres, se identifica una desconexión clara entre los tomadores de decisiones y el documento maestro que describe las amenazas, la vulnerabilidad y el riesgo (Menzel, 2009, p. 120). El documento se centra en las causas y características de las amenazas, individuos o comunidades vulnerables, mas no en los instrumentos y capacidades institucionales para hacer frente a los desastres, donde los instrumentos de planificación, como el Atlas, deben estar orientados a reconocer las capacidades y resiliencia ante los desastres en temas emergentes (Rodríguez, 2015).
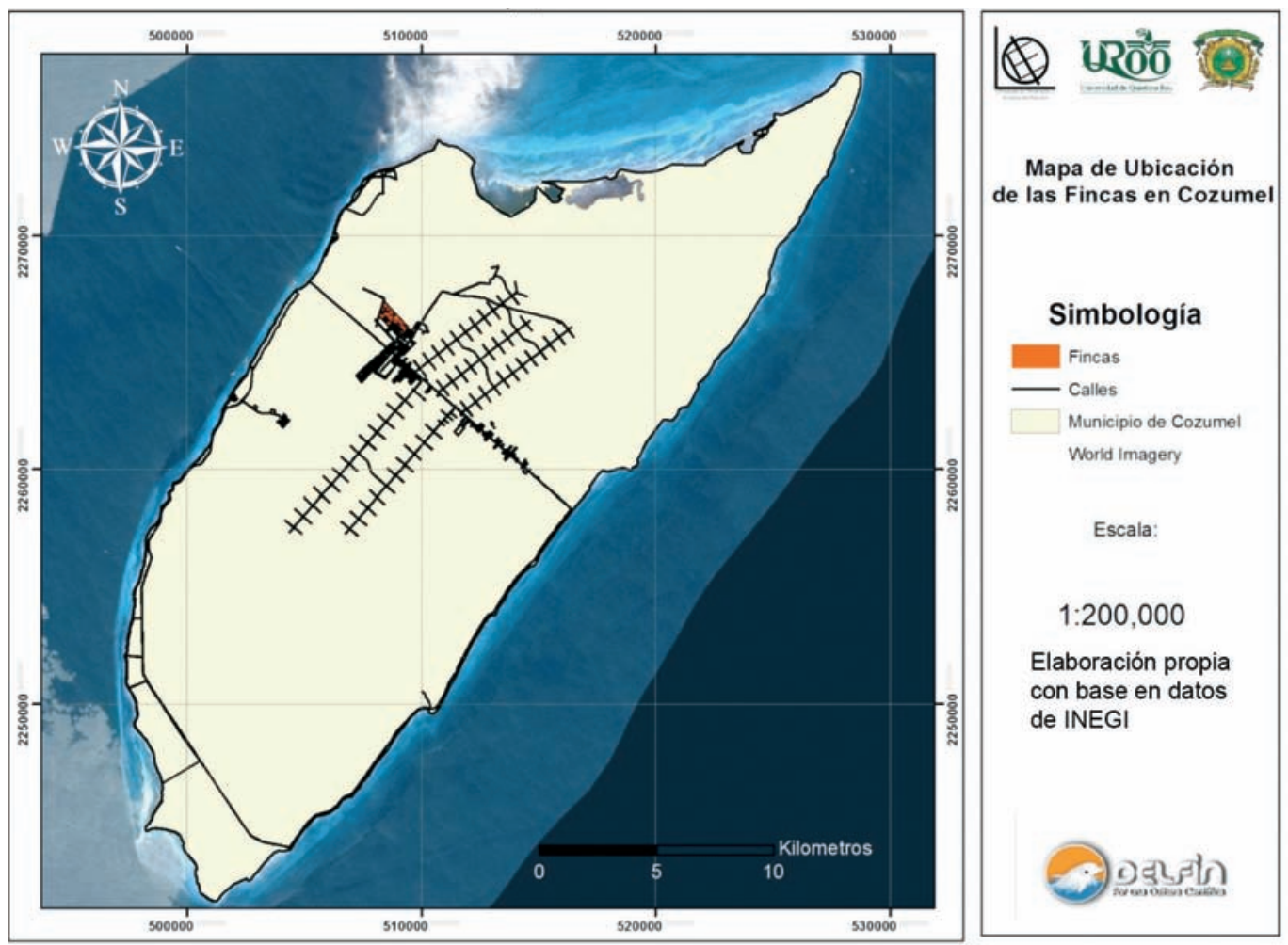

Fuente: Elaboración propia.

Figura 6. Asentamiento irregular Las Fincas en el municipio de Cozumel 


\section{Atlas de Riesgos Cozumel 2011}

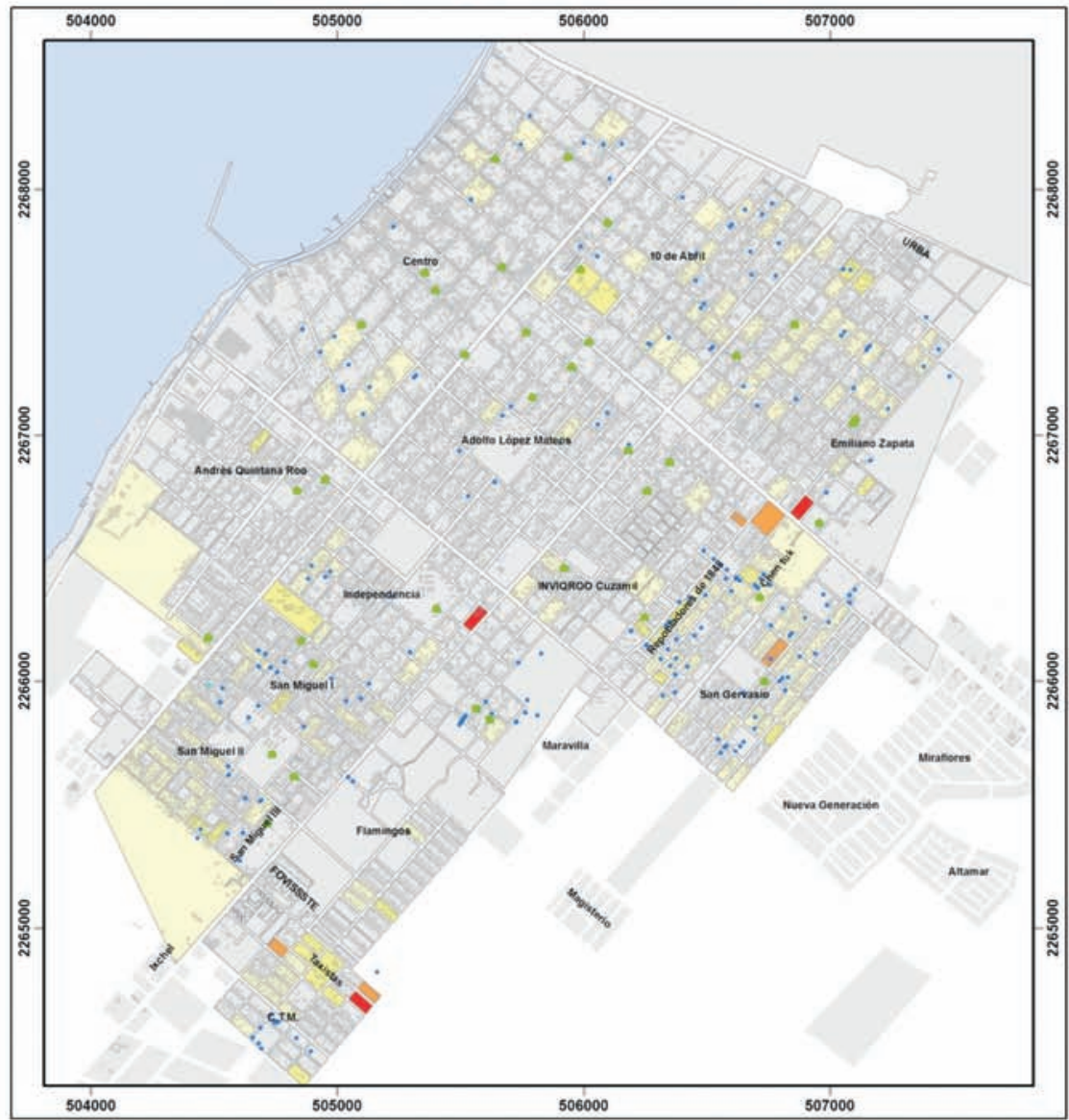

Mapa de Riesgo: Destrucción de las viviendas endebles por los vientos
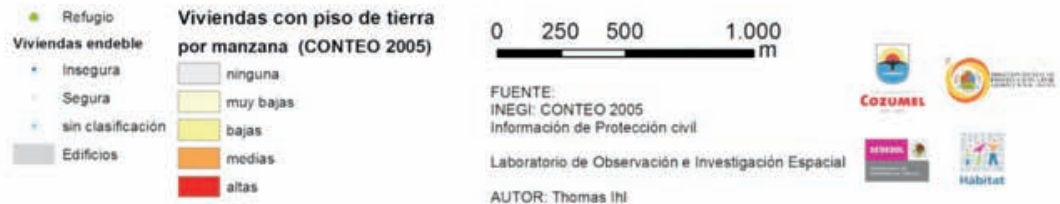

Fuente: Elaboración propia.

Figura 7. Viviendas endebles en Cozumel 


\section{Conclusiones}

La actualización del Atlas de Riesgos de la Isla de Cozumel se realiza con base en los lineamientos de la Ley General de Protección Civil y las guías metodológicas para la elaboración del Atlas de Riesgo Municipal del Cenapred. Si bien existe un atlas antecedente (de 2004, con información de referencia del año 2000 y con una escala de resolución a 1:250000), se busca consolidar uno en función de cinco premisas:

a) El reconocimiento de un punto de partida, a través de los daños ocasionados por los huracanes Emily y Wilma en 2005.

b) El relevamiento catastral de 2008 y 2011.

c) Bases topográficas y de percepción remota a escala detallada (usando modelos del terreno LiDAR y derivados a escala 1:4500).

d) Un sistema de bases de datos georreferenciado a escala detallada, con levantamiento de información base para el modelado de los escenarios de amenaza, vulnerabilidad y riesgo para 2011.

e) Generación de materiales cartográficos solicitados ex profeso por miembros del Comité Directivo de Protección Civil Municipal.

A partir de 2014 se emitió el documento maestro Bases para la Estandarización en la Elaboración de Atlas de Riesgos y Catálogo de Datos Geográficos para representar el Riesgo 2014, de la Secretaría de Desarrollo Agrario, Territorial y Urbano, que busca estandarizar y, a su vez, enriquecer los elementos mínimos que debe considerar un atlas, lo cual se deriva del aprendizaje de la cartografía de los desastres desde principios de los noventa.

En el documento maestro del Atlas se reconoce el principio de reducción del riesgo a desastre, por medio de una serie de recomendaciones y proyectos para la mitigación de escenarios y acciones a corto y mediano plazo.

\section{Agradecimientos}

El presente estudio se desprende del proyecto Diagnóstico y Homogeneización de Bases de Datos Georreferenciadas para el Monitoreo de Riesgo y Desastres Asociados a Fenómenos Hidrometeorológicos y Climáticos en la Península de Yucatán, apoyado por 
la Red de Desastres Hidrometeorológicos y Climáticos (REDESClim) del Consejo Nacional de Ciencia y Tecnología (Conacyt), y Atlas de Riesgos del Municipio de Cozumel, Quintana Roo, apoyado por el H. Ayuntamiento de Cozumel y el programa Hábitat de la Sedesol.

\section{Fuentes consultadas}

Birkmann, J. (2006). Measuring vulnerability to promote disaster-resilient societies: Conceptual frameworks and definitions. En J. Birkmann (ed.), Measuring Vulnerability to Natural Hazards: Towards Disaster Resilient Societies (pp. 9-54). Hong Kong: United Nations University Press.

Castillo-Villanueva, L. y Velázquez-Torres, D. (2015). Sistemas complejos adaptativos, sistemas socio-ecológicos y resiliencia. Quivera, 17(2), 11-32.

Colín, O., Chimal, A. e Ihl, T. (2015). Fenómenos hidrometeorológicos extremos en ciudades turísticas del norte de Quintana Roo. Teoría y Praxis, núm. esp., 84-98.

Colín, O., Frausto, O., Jiménez, J. y García, B. (2015). Aplicación de los Sistemas de Información Geográfica para la gestión sustentable de playas y costas en Cozumel, México. Academia Journals, 5(3), 648-652.

Dirección de Desarrollo Urbano. (2010). Plan de Catastro Municipal 2008. Cozumel: H. Ayuntamiento de Cozumel.

Fernández, A., Romero, R. y Zavala, J. (2014). Bases de datos georreferenciadas en la Unidad de Informática para las Ciencias Atmosféricas y Ambientales (UNIATMOS) del Centro de Ciencias de la Atmósfera de la Universidad Nacional Autónoma de México. En O. Frausto (coord.), Monitoreo de riesgo y desastre asociados a fenómenos hidrometeorológicos y cambio climático (pp. 24-34). Cozumel: Universidad de Quintana Roo/REDESClim-Conacyt.

Frausto, O., Ihl, T., Rojas, J. y Morales, N. (2014). Atlas de amenazas, peligro y vulnerabilidad de Cozumel. Cozumel: Universidad de Quintana Roo.

Frerks, G., Warner, J. y Weijs, B. (2011). The politics of vulnerability and resilience. Ambiente \& Sociedade, XIX(2), 105-122.

H. Ayuntamiento de Cozumel. (2004). Estudio Mapa de Riesgo en Diferentes Polígonos del Municipio de Cozumel. Cozumel: H. Ayuntamiento de Cozumel. 
H. Ayuntamiento de Cozumel. (2012). Atlas de Riesgos del Municipio de Cozumel. Cozumel: Ayuntamiento Municipal de Cozumel.

Hernández-Aguilar, M. L. y Castillo-Villanueva, L. (2012). Capacidad institucional ante la reducción del riesgo de desastres en Quintana Roo: Marco de Acción de Hyogo. Quivera, 14(2), 23-48.

Ihl, T., Frausto, O., Tun, J., Izquierdo, C. y Rolfes, M. (2009). Principios para la elaboración del Atlas de incidencia delictiva de las principales ciudades de Quintana Roo. Teoría y Praxis, 6, 95-105.

INEGI. (2008). Ortofotos de la ciudad de Cozumel a escala 1:4500. México: Instituto Nacional de Estadística y Geografía.

INEGI. (2009). Datos de nivel terreno del modelo LiDAR. México: Instituto Nacional de Estadística y Geografía.

InEgi. (2010). Censo de Población y Vivienda 2010. México: Instituto Nacional de Estadística y Geografía.

INEGI. (2015). Encuesta Intercensal 2015. México: Instituto Nacional de Estadística y Geografía.

Menzel, S. (2009). Vulnerability and Resilience in Natural Disasters: A Marketing and Public Policy Perspective. Journal of Public Policy \& Marketing, 28(1), 114-123.

Mota, L. y Frausto, O. (2015). Environmental education for tourism providers and volunteers in Marine Protected Areas: The case of the National Park Cozumel Reef. Turismo \& Desemvolvimento, 24, 133-145.

Palacio, Á. (2014). El uso de los atlas en el estudio regional y local de los peligros de origen natural en Campeche. En O. Frausto (coord.), Monitoreo de riesgo y desastre asociados a fenómenos hidrometeorológicos y cambio climático (pp. 50-56). Cozumel: Universidad de Quintana Roo/ REDESClim-Conacyt.

Rodríguez, D. (2015). Vulnerabilidad y riesgo. En C. Gay, A. Cos y C. T. Peña (eds.), Reporte Mexicano de Cambio Climático. Grupo II. Impactos, vulnerabilidad y adaptación (pp. 257-279). México: Programa de Investigación en Cambio Climático-Universidad Nacional Autónoma de México.

Rojas, J. y Frausto, O. (2014). Vulnerabilidad a inundaciones con base en el método NBI en el cono sur de Yucatán. En O. Frausto (coord.), Moni- 
toreo de riesgo y desastre asociados a fenómenos hidrometeorológicos y cambio climático (pp. 58-63). Cozumel: Universidad de Quintana Roo/ REDESClim-Conacyt.

Ruiz, I., Frausto, O. y Velázquez, E. (2015). Precariedad de la vivienda en asentamientos irregulares en los destinos turísticos: Cozumel, Quintana Roo, México. Investigación y Posgrado, 5(17), 36-47.

Sánchez, R. y Cavazos, T. (2015). Amenazas naturales, sociedad y desastres. En T. Cavazos (ed.), Conviviendo con la naturaleza: El problema de los desastres asociados a fenómenos hidrometeorológicos y climáticos en México (pp. 1-45). México: REDESClim-Conacyt.

Sedatu. (2014). Bases para la Estandarización en la Elaboración de Atlas de Riesgos y Catálogo de Datos Geográficos para representar el Riesgo 2014. México: Secretaría de Desarrollo Agrario, Territorial y Urbano-Gobierno de la República.

Shi, P., Wang, J., Liu, L., Zhang, W., Chen, W. y Lv, L. (September, 2009). Compilation of Disaster Risk Atlas and its Application in Integrated Disaster Risk Governance. In Expert Meeting of 2nd OECD Catastrophe Risk Management Conference. Bangkok, Thailand.

Vivas, V. (2006). Devastación: Wilma, el azote de Cozumel. México: H. Ayuntamiento de Cozumel. 